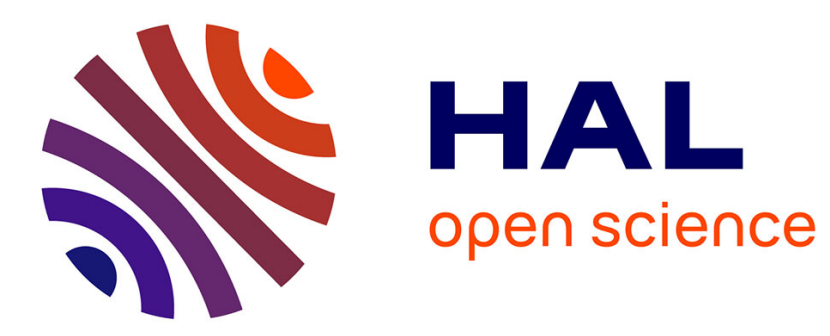

\title{
Time delay measurements: estimation of the error budget
}

G Rovera, M Siccardi, S Römisch, M Abgrall

\section{To cite this version:}

G Rovera, M Siccardi, S Römisch, M Abgrall. Time delay measurements: estimation of the error budget. Metrologia, 2019, 56 (3), pp.035004. 10.1088/1681-7575/ab14bb . hal-03130514

\section{HAL Id: hal-03130514 \\ https://hal.science/hal-03130514}

Submitted on 3 Feb 2021

HAL is a multi-disciplinary open access archive for the deposit and dissemination of scientific research documents, whether they are published or not. The documents may come from teaching and research institutions in France or abroad, or from public or private research centers.
L'archive ouverte pluridisciplinaire HAL, est destinée au dépôt et à la diffusion de documents scientifiques de niveau recherche, publiés ou non, émanant des établissements d'enseignement et de recherche français ou étrangers, des laboratoires publics ou privés. 
Metrologia

PAPER • OPEN ACCESS

Time delay measurements: estimation of the error budget

To cite this article: G D Rovera et al 2019 Metrologia 56035004

View the article online for updates and enhancements. 


\title{
Time delay measurements: estimation of the error budget
}

\author{
G D Rovera ${ }^{1}$, M Siccardi $^{2}$, S Römisch ${ }^{3}$ and M Abgrall ${ }^{1}$ \\ ${ }^{1}$ LNE-SYRTE, Observatoire de Paris, Université PSL, CNRS, Sorbonne Université, 61 avenue de \\ l'Observatoire 75014 Paris, France \\ 2 SKK Electronics Via XX Settembre 30, Cuneo, Italy \\ ${ }^{3}$ NIST Time and Frequency Division, 325 Broadway St. Boulder, CO 80305, United States of America \\ E-mail: daniele.rovera@obspm.fr
}

Received 4 December 2018, revised 22 February 2019

Accepted for publication 29 March 2019

Published 16 May 2019

\begin{abstract}
The measurement of the time difference between two heterogeneous electrical sources is a necessary task in several branches of metrology. In particular the dissemination of Coordinated Universal Time (UTC) around the world requires one to quantify the synchronization between two or more co-located time scales. The task is usually carried out using a time interval counter, by directly measuring the time difference between signals at its inputs. We found that special care must be taken when a total uncertainty significantly smaller than $1 \mathrm{~ns}$ is desired. The goal of this paper is to present a complete analysis of the total uncertainty for the measurement of time differences between co-located time scales, with considerations for improvement.
\end{abstract}

Keywords: pulsed signals, time difference, uncertainty budget, rise time, jitter, time interval counter

(Some figures may appear in colour only in the online journal)

\section{Introduction}

The measurement of the time difference between two timing sources requires a clear definition of the quantities involved. Timing information is usually associated with given events happening in a signal produced by each timing source. These events are called 'time markers' (TM). In our work, we will deal with TM defined as two-level electrical signals. The measurement of the time difference between two TM is usually performed by a time interval counter (TIC).

This measurement is affected by both type A and type B uncertainties [1]. We restrict the scope of this work to the comparison of two co-located and coherent sequences of TM, realized by one-pulse-per-second (1PPS) signals differing by less than a few microseconds.

Additionally we will not consider measurements of the rate of the 1PPS signals. In other words, we are not interested

(i) Original content from this work may be used under the terms of the Creative Commons Attribution 3.0 licence. Any further distribution of this work must maintain attribution to the author(s) and the title of the work, journal citation and DOI. in the time interval between two consecutive events of the same time series. Instead, we will discuss the time difference between any two (heterogeneous) time series [2], at their reference points.

Finally, we will assume that the two series of TM are issued from two similar low-noise clocks nominally at the same frequency. In the case where the two clocks have a small and stable frequency difference, the time delay is no longer a constant. But it can be estimated as a function of time, if the relative time drift is predictable. The measurement is just a little bit more complicated but feasible. The total uncertainty analysis will be similar to the one with clocks of the same frequency, with an additional bias term $b_{t d}$ not considered here, and related to the knowledge of the relative time drift.

By losing some generality, we assume that the timing events on both time scales are similarly defined. This allows one to use a measurement technique called 'The Pivot'. This method consists of a differential measurement that rejects several sources of uncertainty, although not all, as it will be discussed later on. 


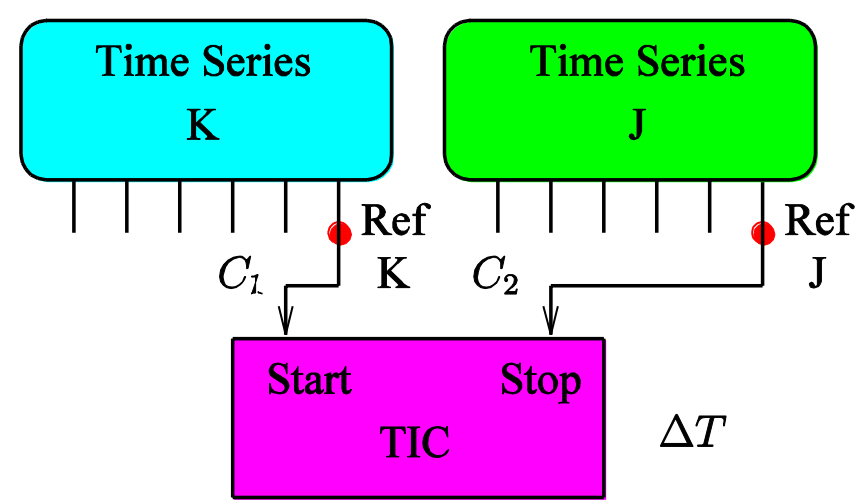

Figure 1. Illustration of setup for a direct measurement of the time difference between time series $J$ and $K$. There is no mitigation of the biases introduced by the TIC and prior knowledge of the time delay introduced by the cables is needed.

\section{Definition of the measurand}

In this paper, we define TM of 1PPS signals as the instants when the rising edge of the pulsed electrical signal crosses a voltage threshold level, at a well defined reference plane, and when properly terminated. The rise time is conventionaly defined as the time required for the signal to go from $10 \%$ to $90 \%$ of its total transition [3].

A typical 1PPS signal raises nominally between $0 \mathrm{~V}$ and $2.5 \mathrm{~V}$ when terminated on a $50 \Omega$ load. Other 1PPS signals rise to $3.5 \mathrm{~V}$, or $5 \mathrm{~V}$, on a $50 \Omega$ load. The output impedance of 1PPS sources is generally $50 \Omega$, but in some cases it might be smaller. Many laboratories set the TM [4] at $1 \mathrm{~V}$ threshold level, but others prefer $1.25 \mathrm{~V}$ or half of the 1PPS amplitude. We choose a threshold voltage of $1.0 \mathrm{~V}$, while the amplitude of the 1PPS can be any of the above, but always terminated in a $50 \Omega$ load.

The reference plane is chosen at the output connector of the device that generates the 1PPS signal of the time scale. This is also called the reference point of the time series. The exact position of the reference plane within the connector is not critical, since $1 \mathrm{~mm}$ of coaxial transmission line introduces a delay of about $5 \mathrm{ps}$, which is small with respect to accuracy targets in the order of tens or hundreds of picoseconds. However, when a better accuracy is expected, a genderless RF coaxial connector like the APC-7, with a well defined geometry of the interface, can be used.

The time difference is defined at the reference planes of the time series, but these reference planes are generally not directly reachable by the TIC, for several reasons including security, reliability of the connections, proximity of the apparatus generating the two time series, common sense, and so on. In practice, the signals are transferred to the TIC by coaxial cables, adding delays that must be estimated with the appropriate uncertainty.

\section{Measurement techniques}

The simplest way to measure the time difference between two time series is to connect their reference points to a TIC using two coaxial cables as shown in figure 1. This simple setup
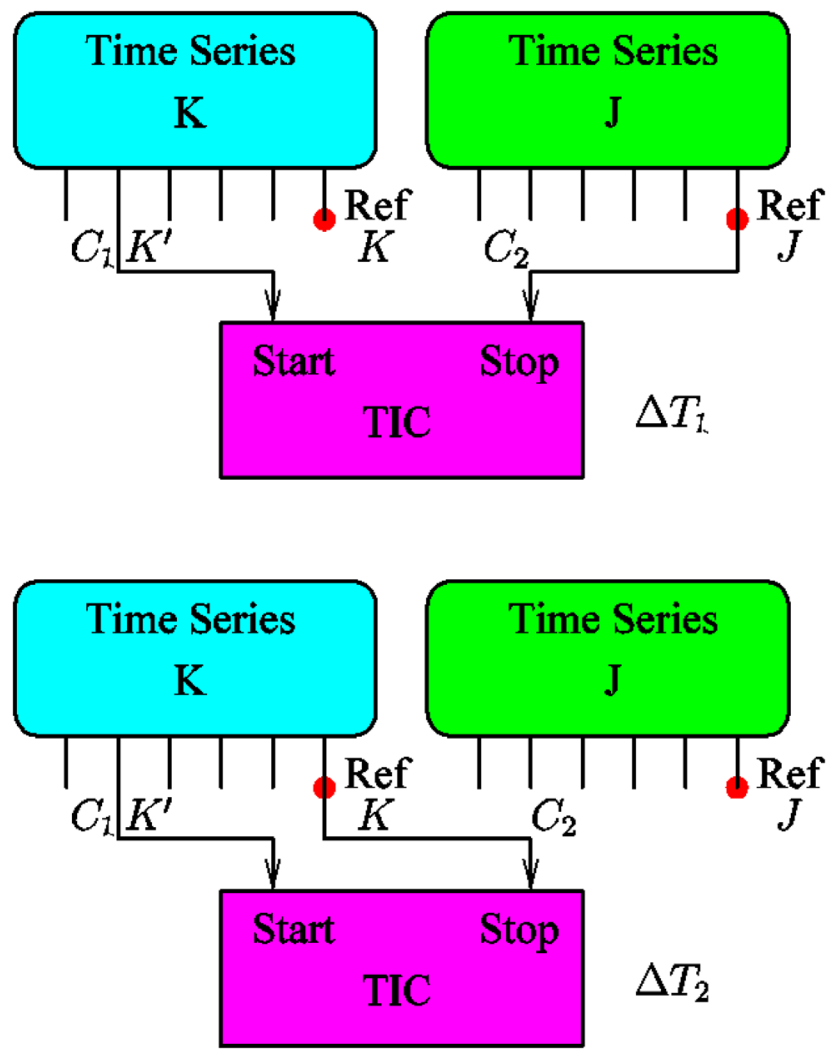

Figure 2. Illustration of the differential measurement technique called pivot. Cable $C 1$ is connected to the pivot, while cable $C 2$ is successively connected to the reference point of time series $J$ and $K$. The difference between the measurements $\Delta T_{1}$ and $\Delta T_{2}$ provides the time difference between the two time series. Some uncertainties introduced by the TIC are mitigated, because they are common to both measurements, and no prior knowledge of the cables delays is needed.

requires the knowledge of the electrical length (time delay) of these two cables, and of all the biases introduced by the TIC. Moreover, if the prior measurement of the cable delays was performed using a vector network analyzer and a sinusoidal signal, or using pulses that have substantially different rise times, the resulting measured delays are biased with respect to the delay actually experienced by the pulses provided by time series $J$ and $K$, introducing a bias in the measured time difference that depends on the characteristics of the pulse and of the cables [5].

The pivot technique, illustrated in figure 2, consists of differential measurements that allow for the cancellation or reduction of part of those biases.

An arbitrary pivot ( $K^{\prime}$ in this example) is chosen and connected to the start port of a TIC using cable $C 1$. The stop port of the TIC is successively connected to the reference point of time series $J$ and time series $K$ using the same cable $C 2$. The difference between the two measurements $\left(\delta T_{1}\right.$ and $\left.\delta T_{2}\right)$ provides the time difference between time series $J$ and $K$, which is the quantity of interest. The technique largely cancels the biases introduced by the TIC, because they are common to both measurements. Moreover, no prior knowledge of the time delay introduced by the cables is needed because it cancels out when calculating the difference. 
In particular, the results of the two measurements shown in figure 2 are

$$
\begin{aligned}
\Delta T_{1} & =(J+C 2)-\left(K^{\prime}+C 1\right)+b_{J K^{\prime}} \pm u_{1} \\
& =J-K^{\prime}+C 2-C 1+b_{J K^{\prime}} \pm u_{1} \\
\Delta T_{2} & =(K+C 2)-\left(K^{\prime}+C 1\right)+b_{K K^{\prime}} \pm u_{2} \\
& =K-K^{\prime}+C 2-C 1+b_{K K^{\prime}} \pm u_{2}
\end{aligned}
$$

where $C_{1}$ and $C_{2}$ represent the time delays of the two cables used in the setup and $u_{1}$ and $u_{2}$ are the uncertainties associated with each measurement.

As will be described in detail later, in addition to the uncertainty of the measurements, there are also a number of biases $\left(b_{J K^{\prime}}\right.$ and $b_{K K^{\prime}}$ ), due to the possible dissimilarity between pulses from time series $J$ and $K$, differently affected by cable dispersion and the limited bandwidth of TICs. So in the end, the time difference between time series $J$ and $K$ is

$$
J-K=\Delta T_{1}-\Delta T_{2}+b \pm u
$$

where $b=b_{J K^{\prime}}-b_{K K^{\prime}}$ is the residual bias, and $u=f\left(u_{1}, u_{2}\right)$ where the function is discussed in section 5 .

If the time series $J$ and $K$ have pulses generated by virtually identical equipment, the residual bias $b$ is zero. That is because the two biases $b_{J K^{\prime}}$ and $b_{K K^{\prime}}$ cancel out due to the pivot method. Nevertheless, in acknowledgment of the non-ideal conditions of any experimental setup, we assume a minimum residual bias of $5 \%$ even when the pulses are generated by virtually identical equipment. This arbitrary value is intended for reference only, and represents the expected difference in between similar electronic components and equipment. A smaller figure can be used, when supported by observation and/or by modeling.

\section{Sources of uncertainty}

In this section we introduce the main sources of uncertainty to be considered in the error budget for time difference measurements as defined in section 2. For biases that can be applied as a correction to the measurement result, only the uncertainty of the bias will be taken into account. If the bias cannot be estimated, and is not compensated by the pivot technique, it will be included in the final uncertainty. We consider first the sources of uncertainty that do not depend on the rise time of the pulses. These include the time jitter, TIC quantization error, the TIC time-base error, and TIC non linearity.

Subsequently we study the sources of uncertainty that depend on the rise time of the pulses. These include the TIC trigger level error, impedance mismatch, the delay of the TIC input filter, and test cables delays.

To illustrate the sources of uncertainty we present the error budget for three different TICs, named in the following as TIC A, TIC B and TIC C.

\subsection{TIC quantization error and jitter}

Some manufacturers specify the TIC resolution as a combination of the quantization error and the time base jitter. We
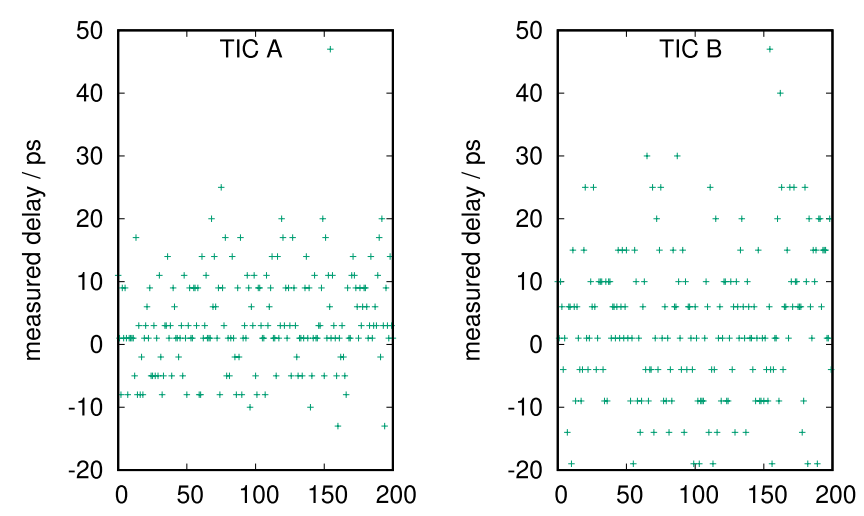

Figure 3. Measurement of a constant noisy delay showing the quantization levels of TICs A and B over 200 samples.

prefer to separate these components, and consider the quantization error alone. We then combine the time-base jitter and the jitter of the measurand in the type-A uncertainty, since they are indistinguishable.

We estimate the quantization error of the TICs experimentally, by measuring a constant delay with a jitter larger than the expected quantization error of the TICs. Irrespective of the specific statistical properties of the noisy source jitter, the effect of quantization on the measurements can be seen in figure 3, where the quantization levels are spaced by no more than 4 ps for the TIC A and no more than 5 ps for the TIC B. The TIC C produces data with 1 fs resolution with no visible quantization.

The uncertainty associated with the quantization is a type B uncertainty that in an ideal case can be estimated as $\delta t / \sqrt{12}[1]$, where $\delta t$ is the quantization error. We retain for both the TICs A and B a value of 2 ps, while for the TIC C we consider $1 \mathrm{ps}$, that is the minimum nonzero value in our computation.

\subsection{TIC time-base error}

The time-base error is proportional to the measured time difference and to the frequency error of the time base. It can be expected that a TIC has an internal quartz frequency oscillator with a relative frequency error of about $10^{-6}$. This might be one order of magnitude better when the device is freshly calibrated or if a high-stability option was purchased, and at least two orders of magnitude better when a rubidium time base is present. In order to produce high quality measurements, the TIC must be disciplined to an accurate external frequency reference. In a time metrology laboratory equipped with a high quality frequency standard, i.e. a commercial Cs or a $\mathrm{H}$ maser, the external time-base relative accuracy can be at least $10^{-12}$ or better. Under these conditions, when measuring a time interval smaller than $1 \mathrm{~s}$, the time base error is smaller than 1 ps and can be neglected. However, if the TIC time-base is a Rubidium frequency standard with a typical frequency error of $10^{-9}$, which is the best case of an unreferenced TIC, the error can be of the order of $1 \mathrm{~ns}$ when reading a $1 \mathrm{~s}$ time delay. Differential measurement techniques like the pivot technique do not mitigate this error. 


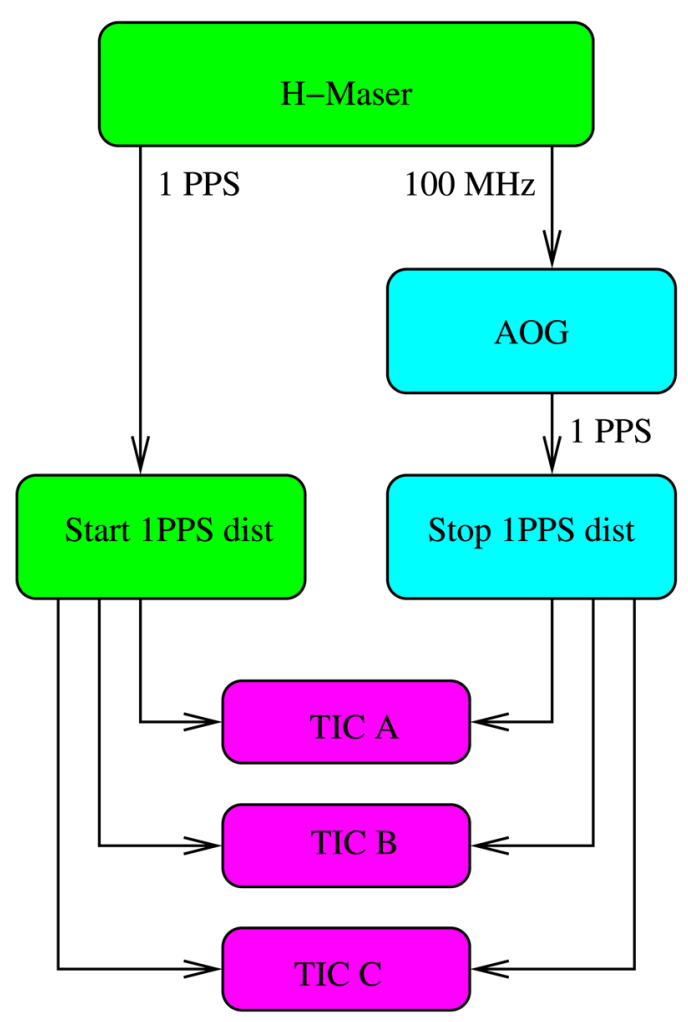

Figure 4. Setup used to measure the non linearity of the three TICs.

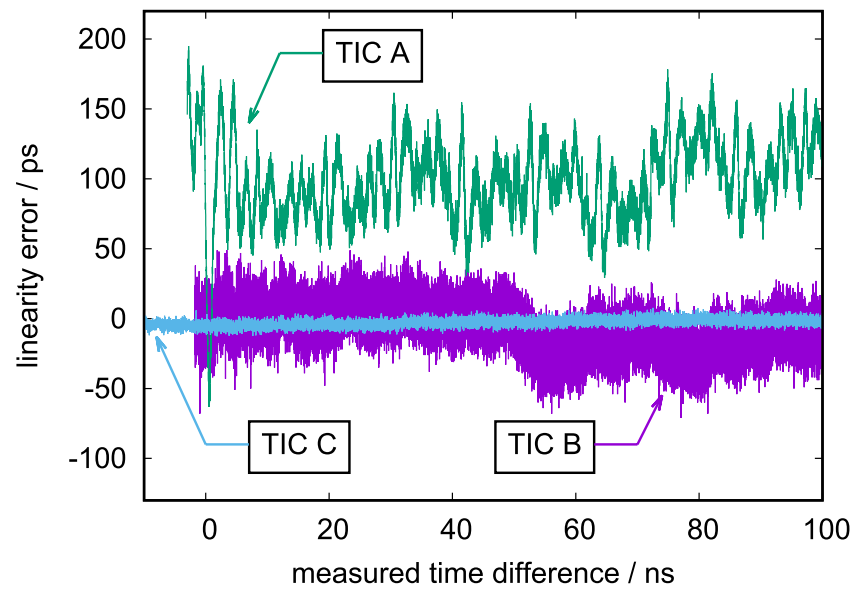

Figure 5. Non-linearity error of the three TICs considered: violet TIC B, green TIC A, light-blue TIC C. An arbitrary offset has been introduced for clarity.

\subsection{TIC non-linearity}

Measurements with TICs can be affected by a deterministic error that depends on the measured value, due to the nonlinear behavior of the interpolator: we call this contribution the non-linearity error. All the TICs considered here use an interpolator to enhance their resolution. Several interpolation techniques can be used [6], but all of them are affected by some non-linearity error. Non linearity errors have been evaluated experimentally using the setup shown in figure 4 , where an AOG (auxiliary offset generator) [7] produces a series of pulses, sent to the stop input of the TICs, with a known rate offset with respect to the time series going directly to the start

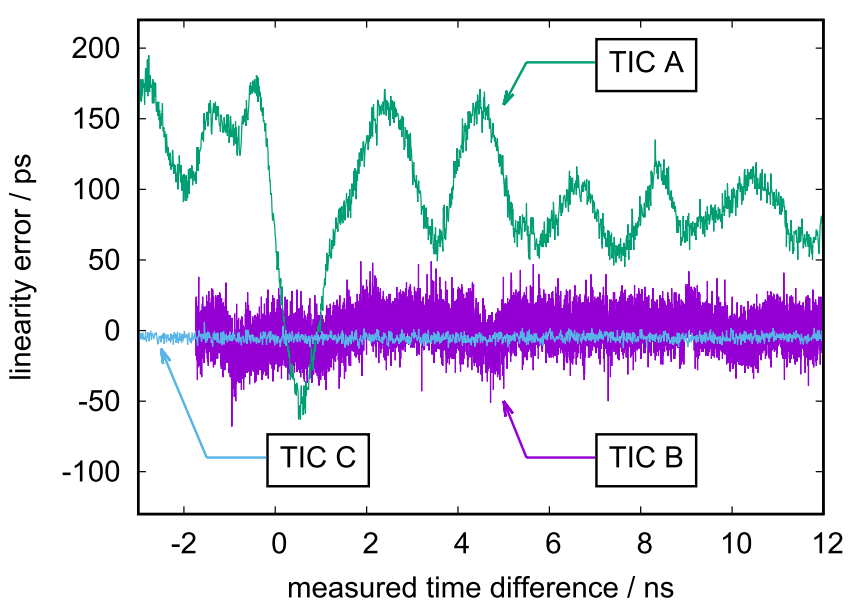

Figure 6. TIC non linearity for small time intervals: violet TIC $\mathrm{B}$, green TIC A, light-blue TIC C. An arbitrary offset has been introduced for clarity.

input of the TICs. The three TICs therefore measure a time difference that grows linearly in time.

Figures 5 and 6 show the differences between the measurements and the expected values computed after removing the known rate offset introduced by the AOG, for the three different instruments. In both figures an arbitrary offset has been added to the green curve (TIC A) for clarity.

The largest non-linearity is found in the data from TIC A, which is based on an analog interpolator developed in the 1980s. When the TIC is measuring time intervals larger than $10 \mathrm{~ns}$ the measured non-linearity is compatible with the 50 ps non-linearity declared by the manufacturer, but when measuring shorter time intervals the non-linearity error can be two to three times larger. To be sure that this is not a problem specific to the individual unit, we have repeated the experiment with other units of the same model, and obtained similar results. The behavior of TIC A can likely be explained by the cross-talk between the two input channels that impacts the measured value when the pulses on them are closer in time.

The more modern TIC B has a smaller more uniform nonlinearity error that is in line with the claimed accuracy of 100 ps. By smoothing out the noise with a moving average, we can deduce that the residual non-linearity is 40 ps peak to peak. Considering a rectangular probability distribution the standard uncertainty [1] is estimated to be $u_{\mathrm{int}}=40 / \sqrt{(12)}=12 \mathrm{ps}$.

For the TIC C we found no evidence of non-linearity at the ps level.

\subsection{TIC Trigger error}

The uncertainty of the trigger level leads to a type B timing uncertainty proportional to the inverse of the 1PPS slew rate. In a typical case of a slow-rising 1PPS signal with a slope of $0.7 \mathrm{~V} \mathrm{~ns}^{-1}$, an uncertainty of $20 \mathrm{mV}$ in the trigger level leads to a timing uncertainty of $28 \mathrm{ps}$. A fast-rising pulse with a slew-rate of $7 \mathrm{~V}$ per ns, the same uncertainty of $20 \mathrm{mV}$ on the trigger level generates a timing uncertainty of approximately 3ps. The hysteresis is usually acting dynamically on the signal according to the edge polarity. Its contribution is 
contained within the trigger level uncertainty provided by the manufacturer.

The trigger error is fully compensated when the pivot method is used to measure the time difference between two signals with identical pulses. Although unknown, the trigger error is expected to be the same for all measurements performed at the same threshold level, therefore if the signals have the same slope, the error is assumed to be the same. As discussed in section 3 even when the pulses are considered identical we assume that $5 \%$ of the actual bias is not compensated by the pivot technique. When pulses have different slopes, the pivot method will not cancel this error.

\subsection{TIC impedance mismatch}

Our definition of TM states for the load impedance to be at a nominal value of $50 \Omega$. In case of deviation of the load impedance from its nominal value, the pulse amplitude is affected like in a voltage divider, so that the error induced by an impedance mismatch can be considered in the same way as a trigger error.

In the examples described in this paper we consider devices with, in the worst case, a real input impedance of $(50 \pm 5)$ $\Omega$, corresponding to a VSWR (voltage standing wave ratio) of 1.1. We estimate the timing error due to this amount of impedance mismatch to be approximately 70 ps for slowrising pulses (slew rate of 0.7 Vper ns) and 7 ps for fast-rising pulses (slew rate of $7 \mathrm{~V}$ per ns). As for trigger errors, the pivot method allows for a compensation of the bias only if the measured 1PPS outputs are identical. In the ideal case the output impedance of the 1PPS generator has no direct influence on the measurement result, nevertheless in some cases it can reduce the compensation introduced by the pivot method. We consider here that the output impedance of both devices is similar enough that the resulting bias can be included within the residual $5 \%$ uncertainty we retain for the cancellation introduced by the pivot method.

\subsection{TIC input filter}

To avoid false triggering and to improve the stability when the TICs are operating in frequency measurement mode, the front ends of commercial counters usually include low-pass filters, with bandwidths at the declared maximum measurable frequency or even at a fraction of it. This filter limits the input bandwidth of the TIC, effectively adding a dispersive element in the measurement system that introduces a delay dependent on the shape of the 1PPS signal. It may also reduce the slope of the signal to the TIC internal comparator, thus increasing the impact of trigger level uncertainty.

A rough measurement of the input bandwidth can be carried out by using stable, fast-rising 1PPS signals and plotting the measured delay as a function of the trigger level. The scan of a fast 1PPS, with a rise time of about $350 \mathrm{ps}$ is presented in figure 7 for the three TICs under test. The measured bandwidth of TIC B is in agreement with the $350 \mathrm{MHz}$ declared by the manufacturer. For TIC A, the manufacturer declares a maximum measurable frequency of $300 \mathrm{MHz}$ without specifying

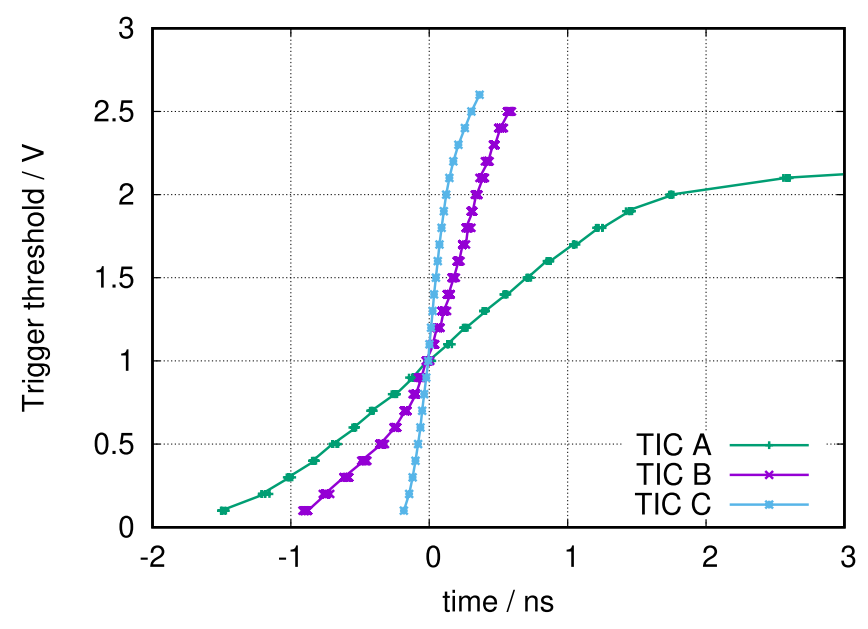

Figure 7. Scan of a fast 1PPS signal characterizing the input filter of tree different counters: violet TIC B, green TIC A, blue TIC C. An arbitrary constant is added to the time axis data in order to align all pulses at the $1 \mathrm{~V}$ trigger point.

an input bandwidth, but indicates reduced sensitivity for frequencies greater than $100 \mathrm{MHz}$. The measured bandwidth is $100 \mathrm{MHz}$. The rise time of about $350 \mathrm{ps}$ resulting from TIC C scan corresponds to the specification of the 1PPS distribution unit and there is no evidence of a bandwidth limitation due to the TIC, whose input bandwidth is declared to be $8 \mathrm{GHz}$ by the manufacturer. The different propagation delays for pulses of different shapes through a low pass filter has been introduced in [5]. Figure 8 shows a simulation of the propagation delay trough a low pass filter as a function of the rise time for various bandwidths. When using the pivot method on signals with similar rising edges the biases introduced by the input filter cancel out.

\subsection{Cable delay}

In time metrology laboratories that utilize the direct measurement technique illustrated in figure 1 it is normal practice to measure the delay of the cables prior to their use, assuming that their actual delay when used in a measurement setup is the value previously measured.

This is acceptable for overall measurement accuracy of a few nanoseconds. If the target accuracy is 100 ps or below, it must be considered that the actual cable delay depends on the shape of the signal propagating through the cable and on the trigger level used by the TIC. Figure 9 reports the numerical simulation of the additional cable delay for three different lengths of the widely used RG58 C/U cable, as a function of the rise time of the pulses propagating through the cables. The cable delay of ideal pulses with negligible rise time is subtracted from the total delay computed for increasing rise times. The plots show the additional cable delay to be considered for rise times larger than 100 ps.

The simulation has been confirmed experimentally by measurements, also reported in figure 9, of the additional delay of two long cables of different lengths. These measurements were performed using TIC $\mathrm{C}$ and therefore were not limited by the TIC bandwidth $(8 \mathrm{GHz})$. 


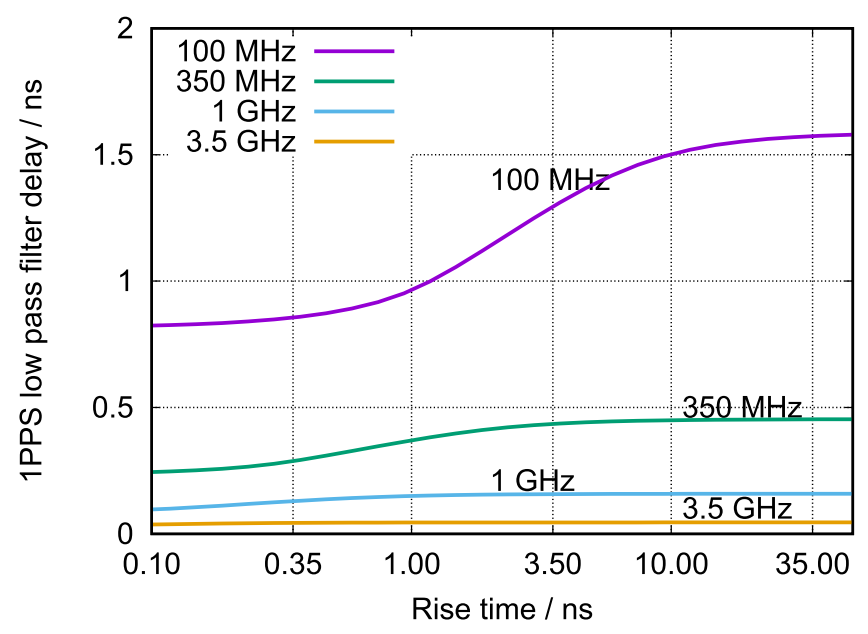

Figure 8. Simulation of the delay of the TM of 1PPS signal passing through different low pass filters.

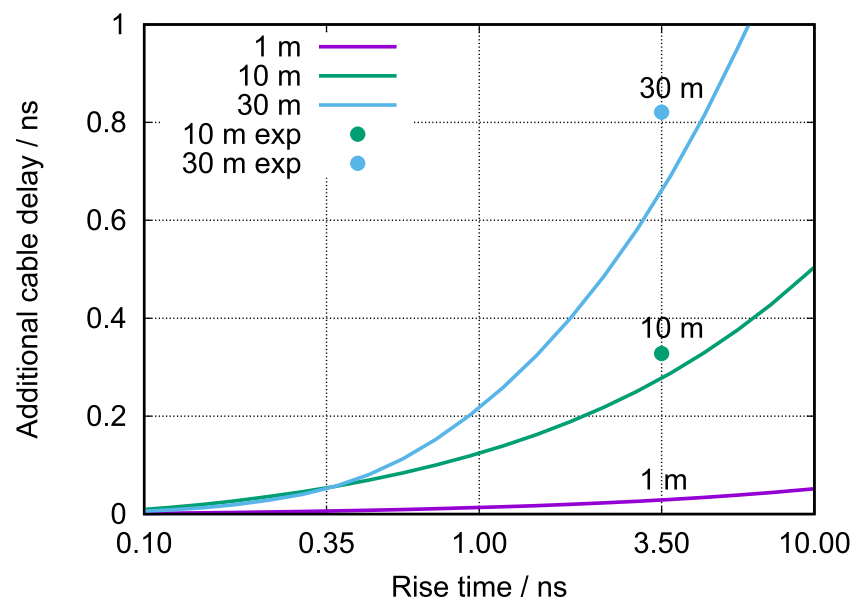

Figure 9. Variation of 1PPS cable delay as a function of rise time for different cable lengths, computed using the model for RG58 C/U cables. The experimental values have been measured with the TIC C.

When measuring the time difference between two sources, the additional delays of the cables, with respect to the ideal case of 100 ps rise time, are canceled out by the pivot method if the rise times of the 1PPS signals are the same. This is not the case when the pulse rise times are different.

\section{Typical error budgets}

In this section we present the total uncertainty of a time difference measurement using the pivot technique, described in section 2 , for two scenarios that are commonly encountered in timing laboratories. In the first scenario, case A, we consider two time series with 1PPS signals with a fast rise time of about $350 \mathrm{ps}$ and $7 \mathrm{~V} \mathrm{~ns}^{-1}$ slew rate as shown in figure 10. In the second scenario, case $\mathrm{B}$, we consider a time series with pulses with a fast rise time as above, and one with a slow rise time of about $3.5 \mathrm{~ns}$ and a $0.7 \mathrm{~V} \mathrm{~ns}^{-1}$ slew rate, as shown in figure 11 .

With reference to figure 2, we will use a $10 \mathrm{~m}$ long, RG58 coaxial cable as cable $C 2$, with a nominal delay of $50 \mathrm{~ns}$ corresponding to a pulse with virtually infinite slew rate (100 ps). To this nominal value, additional delays of 53 ps (for pulses with a rise time of $350 \mathrm{ps}$ ) and $280 \mathrm{ps}$ (for pulses with a rise

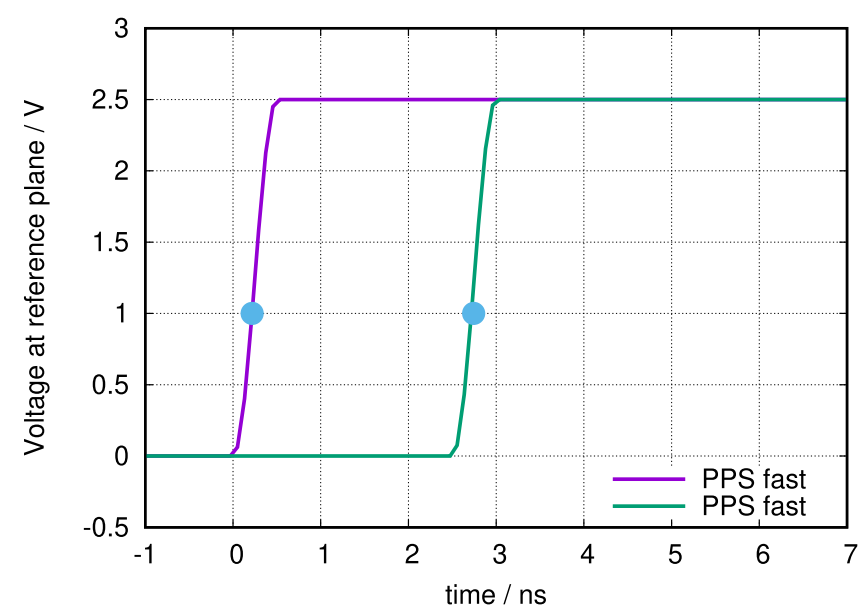

Figure 10. Measurement of time difference between two 1PPS with a fast rise time.

time of $3.5 \mathrm{~ns}$ ) are added as determined by the simulations shown in figure 9.

The total uncertainty analysis for each TIC for both scenarios (Cases A and B) is illustrated in tables 1 through 6. Each line in the tables represents a source of error for time difference measurements, as described in section 4. All presented values are expressed in ps and rounded to the upper integer, as usual in uncertainty budgets. In the first four columns are indicated the expected bias affecting the measurement and its uncertainty, for $\Delta T_{1}$ and $\Delta T_{2}$ as defined in equations (1) and (2).

The last three columns are devoted to the uncertainty of the quantity $J-K$ : the residual bias $b_{(J-K)}$ defined in equation (3), the uncertainty $u_{\text {res }}$ that includes the residual bias and the combination of the measurement uncertainties $u_{1}$ and $u_{2}$ when no correction is applied to eliminate the residual bias $b_{(J-K)}$, and the uncertainty $u_{\text {corr }}$ that can be obtained if the residual bias is evaluated and corrected.

This uncertainty budget may differ from what is presented in a typical TIC operation manual, which is generally more oriented towards the case of measurements performed using the direct technique of figure 1, and where the main source of error is the accuracy of the TIC internal time base. In all cases described in this paper, the TICs were disciplined to accurate external references, rendering this specific source of error negligible.

\subsection{TIC A}

For many years this TIC was the only instrument allowing single shot delay measurement with a resolution of a few ps.

Nowadays this instrument is still one of the most widely used TIC in UTC $(\mathrm{k})$ laboratories all around the world.

From the analysis of figure 5 two different values can be retained for the non-linearity. In the example considered here we assume that TIC A reads values bigger than $10 \mathrm{~ns}$ in all the cases, therefore we adopt the value of 50 ps for the nonlinearity uncertainty.

We begin with the case of two time series with similar fast-rising pulses with a rise time of about 350 ps. For the 


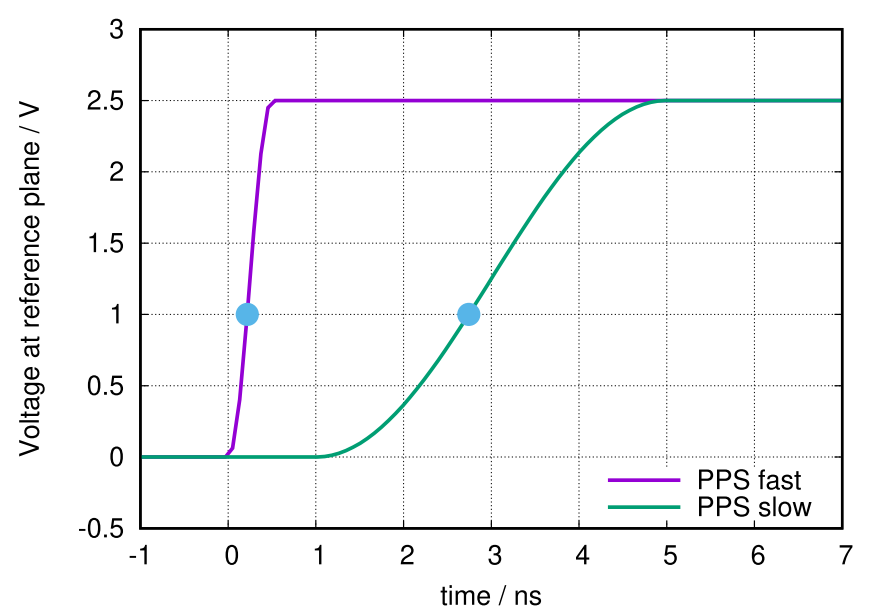

Figure 11. Measurement of time difference between two 1PPS with different rise time.

Table 1. Error budget of the time difference between two similar fast-rising 1PPS (case A) measured with TIC A (all values in ps).

\begin{tabular}{|c|c|c|c|c|c|c|c|}
\hline & \multicolumn{2}{|c|}{$\Delta T_{1}$} & \multicolumn{2}{|c|}{$\Delta T_{2}$} & \multicolumn{3}{|c|}{$(J-K)$} \\
\hline & $b_{K K^{\prime}}$ & $u_{1}$ & $b_{J K^{\prime}}$ & $u_{2}$ & $b_{(J-K)}$ & $u_{\text {res }}$ & $u_{\text {corr }}$ \\
\hline Noise/jitter & 0 & 10 & 0 & 10 & 0 & 14 & 14 \\
\hline $\begin{array}{l}\text { Quantization } \\
\text { error }\end{array}$ & 0 & 2 & 0 & 2 & 0 & 3 & 3 \\
\hline $\begin{array}{l}\text { Time-base } \\
\text { error }\end{array}$ & 0 & 1 & 0 & 1 & 0 & 1 & 1 \\
\hline $\begin{array}{l}\text { TIC non } \\
\text { linearity }\end{array}$ & 0 & 50 & 0 & 50 & 0 & 71 & 71 \\
\hline Trigger error & 0 & 3 & 0 & 3 & 0 & 1 & 1 \\
\hline $\begin{array}{l}\text { Impedance } \\
\text { mismatch }\end{array}$ & 0 & 7 & 0 & 7 & 0 & 1 & 1 \\
\hline $\begin{array}{l}\text { TIC input } \\
\text { filter }\end{array}$ & 856 & 86 & 856 & 86 & 43 & 43 & 43 \\
\hline $\begin{array}{l}\text { Additional } \\
\text { cable delay }\end{array}$ & 53 & 6 & 53 & 6 & 3 & 3 & 3 \\
\hline $\begin{array}{l}\text { Quadratic } \\
\text { sum }\end{array}$ & & & & & & 85 & 85 \\
\hline
\end{tabular}

uncertainty contributions that are uncorrelated, the final uncertainty is the quadratic sum of the uncertainties in the measurement of $\Delta T_{1}$ and $\Delta T_{2}$. For the trigger error and the impedance mismatch, the actual value of the biases can only be estimated (see 4.4 and 4.5). However, the biases are correlated and are compensated by using the pivot method, so their $u_{\text {res }}$ is reduced to $5 \%$ of $u_{1}$ and $u_{2}$.

Finally the biases due to the additional cable delay and the delay of the TIC input filter are as well reduced to $5 \%$ of their value by the use of the pivot method. Their values are numerically evaluated with respect to the delay of a 100-ps rise-time pulse.

When the rise time of the two time series differs, as is Case B, shown in table 2, the time series $J$ has pulses with a rise time of $3.5 \mathrm{~ns}$ (slew rate of $7 \mathrm{v}$ per $\mathrm{ns}$ ). The uncertainty contributions that are different with respect to Case A (similar pulses) are the last four lines in the tables, not fully compensated by the use of the pivot method: trigger error, impedance mismatch, TIC input filter and cable delay. For two of
Table 2. Error budget of the time difference between a fast rising and slow rising 1PPS (case B) measured with TIC A (all values in ps).

\begin{tabular}{|c|c|c|c|c|c|c|c|}
\hline & \multicolumn{2}{|c|}{$\Delta T_{1}$} & \multicolumn{2}{|c|}{$\Delta T_{2}$} & \multicolumn{3}{|c|}{$(J-K)$} \\
\hline & $b_{K K^{\prime}}$ & $u_{1}$ & $b_{J K^{\prime}}$ & $u_{2}$ & $b_{(J-K)}$ & $u_{\text {res }}$ & $u_{\text {corr }}$ \\
\hline Noise/jitter & 0 & 10 & 0 & 10 & 0 & 14 & 14 \\
\hline $\begin{array}{l}\text { Quantiza- } \\
\text { tion error }\end{array}$ & 0 & 2 & 0 & 2 & 0 & 3 & 3 \\
\hline $\begin{array}{l}\text { Time-base } \\
\text { error }\end{array}$ & 0 & 1 & 0 & 1 & 0 & 1 & 1 \\
\hline $\begin{array}{l}\text { TIC non } \\
\text { linearity }\end{array}$ & 0 & 50 & 0 & 50 & 0 & 71 & 71 \\
\hline $\begin{array}{l}\text { Trigger er- } \\
\text { ror }\end{array}$ & 0 & 3 & 0 & 28 & 0 & 25 & 25 \\
\hline $\begin{array}{l}\text { Impedance } \\
\text { mismatch }\end{array}$ & 0 & 7 & 0 & 70 & 0 & 63 & 63 \\
\hline $\begin{array}{l}\text { TIC input } \\
\text { filter }\end{array}$ & 856 & 86 & 1290 & 129 & 434 & 434 & 129 \\
\hline $\begin{array}{l}\text { Additional } \\
\text { cable delay }\end{array}$ & 53 & 6 & 280 & 28 & 227 & 227 & 28 \\
\hline $\begin{array}{l}\text { Quadratic } \\
\text { sum }\end{array}$ & & & & & & 500 & 166 \\
\hline
\end{tabular}

them (trigger error and impedance mismatch) $u_{\text {res }}$ is given by the difference between $u_{1}$ and $u_{2}$, for the last two, $u_{\text {res }}$ is the residual bias $b_{(J-K)}$. In particular, $u_{\text {corr }}$ is then reduced to $u_{2}$ because the residual bias is known and can be corrected.

\section{2. $T I C B$}

Only a few commercial TICs have a single shot resolution in the few ps range, and among them TIC B is one of the most popular after TIC A. The resolution and the time jitter of this TIC are not as good as for TIC A, although they are very similar, but TIC B is more stable over longer times and the its non-linearity is drastically reduced. As shown in figures 5 and 6 a residual non-linearity error of 40 ps peak-to-peak generates an uncertainty of $12 \mathrm{ps}$. The wider input bandwidth allows a reduced uncertainty for the measurement of the time difference of 1PPS signals with different rise time. Concerning the input impedance, the manual of TIC B indicates $50 \Omega \pm 1.5$ $\%$ in parallel with $25 \mathrm{pF}$. In this case the exact evaluation of the mismatch is quite complicated and depends on the harmonic contents of the 1PPS signal. We retain for this TIC an input mismatch uncertainty of $(50 \pm 3) \Omega$, lower than the one considered for TIC A, and at the same level as that of TIC C. The TIC B manual also states a trigger level setting error of $\pm(0.2 \%$-of setting $+0.1 \%$-of range $)$ leading to $7 \mathrm{mV}$ of trigger uncertainty, generating a time uncertainty of $1 \mathrm{ps}$ for fast pulses and $10 \mathrm{ps}$ for the slow ones. Due to the $350 \mathrm{MHz}$ bandwidth the bias due to the input filter is greatly reduced, as shown in figure 8.

\section{3. $T I C C$}

The uncertainty budget estimated for this TIC is a lower limit to the uncertainty of time measurements between 1PPS signals by using commercially available TICs. Table 6 provides 
Table 3. Error budget of the time difference between two similar fast-rising 1PPS (Case A) measured with TIC B (all values in ps).

\begin{tabular}{|c|c|c|c|c|c|c|c|}
\hline & \multicolumn{2}{|c|}{$\Delta T_{1}$} & \multicolumn{2}{|c|}{$\Delta T_{2}$} & \multicolumn{3}{|c|}{$(J-K)$} \\
\hline & $b_{K K^{\prime}}$ & $u_{1}$ & $b_{J K^{\prime}}$ & $u_{2}$ & $b_{(J-K)}$ & $u_{\text {res }}$ & $u_{\text {cor }}$ \\
\hline Noise/jitter & 0 & 15 & 0 & 15 & 0 & 21 & 21 \\
\hline $\begin{array}{l}\text { Quantization } \\
\text { error }\end{array}$ & 0 & 2 & 0 & 2 & 0 & 3 & 3 \\
\hline $\begin{array}{l}\text { Time-base } \\
\text { error }\end{array}$ & 0 & 1 & 0 & 1 & 0 & 1 & 1 \\
\hline $\begin{array}{l}\text { TIC non } \\
\text { linearity }\end{array}$ & 0 & 12 & 0 & 12 & 0 & 17 & 17 \\
\hline Trigger error & 0 & 1 & 0 & 1 & 0 & 1 & 1 \\
\hline $\begin{array}{l}\text { Impedance } \\
\text { mismatch }\end{array}$ & 0 & 4 & 0 & 4 & 0 & 1 & 1 \\
\hline $\begin{array}{l}\text { TIC input } \\
\text { filter }\end{array}$ & 290 & 29 & 290 & 29 & 15 & 15 & 15 \\
\hline $\begin{array}{l}\text { Additional } \\
\text { cable delay }\end{array}$ & 53 & 6 & 53 & 6 & 3 & 3 & 3 \\
\hline $\begin{array}{l}\text { Quadratic } \\
\text { sum }\end{array}$ & & & & & & 32 & 32 \\
\hline
\end{tabular}

Table 4. Error budget of the time difference between a fast rising and slow rising 1PPS (Case B) measured with TIC B (all values in ps) and $0.7 \mathrm{~V} \mathrm{~ns}^{-1}$ slew rate.

\begin{tabular}{|c|c|c|c|c|c|c|c|}
\hline & \multicolumn{2}{|c|}{$\Delta T_{1}$} & \multicolumn{2}{|c|}{$\Delta T_{2}$} & \multicolumn{3}{|c|}{$(J-K)$} \\
\hline & $b_{K K^{\prime}}$ & $u_{1}$ & $b_{J K^{\prime}}$ & $u_{2}$ & $b_{(J-K)}$ & $u_{\text {res }}$ & $u_{\text {cor }}$ \\
\hline Noise/jitter & 0 & 15 & 0 & 15 & 0 & 21 & 21 \\
\hline $\begin{array}{l}\text { Quantiza- } \\
\text { tion error }\end{array}$ & 0 & 2 & 0 & 2 & 0 & 3 & 3 \\
\hline $\begin{array}{l}\text { Time-base } \\
\text { error }\end{array}$ & 0 & 1 & 0 & 1 & 0 & 1 & 1 \\
\hline $\begin{array}{l}\text { TIC non lin- } \\
\text { earity }\end{array}$ & 0 & 12 & 0 & 12 & 0 & 17 & 17 \\
\hline Trigger error & 0 & 1 & 0 & 10 & 0 & 9 & 9 \\
\hline $\begin{array}{l}\text { Impedance } \\
\text { mismatch }\end{array}$ & 0 & 4 & 0 & 40 & 0 & 36 & 36 \\
\hline $\begin{array}{l}\text { TIC input } \\
\text { filter }\end{array}$ & 290 & 29 & 435 & 44 & 145 & 145 & 44 \\
\hline $\begin{array}{l}\text { Additional } \\
\text { cable delay }\end{array}$ & 53 & 6 & 280 & 28 & 227 & 227 & 28 \\
\hline $\begin{array}{l}\text { Quadratic } \\
\text { sum }\end{array}$ & & & & & & 274 & 70 \\
\hline
\end{tabular}

evidence that in the case of pulses of different rise times (Case B) the biggest uncertainty is due to the additional cable delay. When a low uncertainty is crucial the cable length must be reduced to the minimum compatible with the physical locations of the time series. The use of a cable with low dispersion and attenuation is also helpful. In the examples presented here TIC C is used in dual channel start-stop configuration, but this device is quite different from the TICs previously described and can be used as an event timer to time-tag signals of both input channels against the internal time base. This feature is particularly interesting for measurement with the pivot method because the TIC can be used in single channel mode, with each measurement only affected by the noise and the
Table 5. Error budget of the time difference between two similar fast-rising 1PPS (Case A) measured with TIC C (all values in ps).

\begin{tabular}{|c|c|c|c|c|c|c|c|}
\hline & \multicolumn{2}{|c|}{$\Delta T_{1}$} & \multicolumn{2}{|c|}{$\Delta T_{2}$} & \multicolumn{3}{|c|}{$(J-K)$} \\
\hline & $b_{K K^{\prime}}$ & $u_{1}$ & $b_{J K^{\prime}}$ & $u_{2}$ & $b_{(J-K)}$ & $u_{\text {res }}$ & $u_{\text {corr }}$ \\
\hline Noise/jitter & 0 & 2 & 0 & 2 & 0 & 3 & 3 \\
\hline Quantization error & 0 & 1 & 0 & 1 & 0 & 1 & 1 \\
\hline Time-base error & 0 & 1 & 0 & 1 & 0 & 1 & 1 \\
\hline TIC non linearity & 0 & 2 & 0 & 2 & 0 & 3 & 3 \\
\hline Trigger error & 0 & 1 & 0 & 1 & 0 & 1 & 1 \\
\hline Impedance mismatch & 0 & 4 & 0 & 4 & 0 & 1 & 1 \\
\hline TIC input filter & 43 & 5 & 43 & 5 & 2 & 2 & 2 \\
\hline Additional cable delay & 53 & 6 & 53 & 6 & 3 & 3 & 3 \\
\hline Quadratic sum & & & & & & 6 & 6 \\
\hline
\end{tabular}

Table 6. Error budget of the time difference between a fast rising and slow rising 1PPS (Case B) measured with TIC C (all values in ps).

\begin{tabular}{|c|c|c|c|c|c|c|c|}
\hline & \multicolumn{2}{|c|}{$\Delta T_{1}$} & \multicolumn{2}{|c|}{$\Delta T_{2}$} & \multicolumn{3}{|c|}{$(J-K)$} \\
\hline & $b_{K K^{\prime}}$ & $u_{1}$ & $b_{J K^{\prime}}$ & $u_{2}$ & $b_{(J-K)}$ & $u_{\text {res }}$ & $u_{\text {corr }}$ \\
\hline Noise/jitter & 0 & 2 & 0 & 2 & 0 & 3 & 3 \\
\hline Quantization error & 0 & 1 & 0 & 1 & 0 & 1 & 1 \\
\hline Time-base error & 0 & 1 & 0 & 1 & 0 & 1 & 1 \\
\hline TIC non linearity & 0 & 2 & 0 & 2 & 0 & 3 & 3 \\
\hline Trigger error & 0 & 1 & 0 & 1 & 0 & 1 & 1 \\
\hline Impedance mismatch & 0 & 4 & 0 & 4 & 0 & 1 & 1 \\
\hline TIC input filter & 43 & 5 & 45 & 5 & 2 & 2 & 2 \\
\hline Additional cable delay & 53 & 6 & 280 & 28 & 227 & 227 & 28 \\
\hline Quadratic sum & & & & & & 228 & 29 \\
\hline
\end{tabular}

non-linearity of one channel. In this configuration the events are dated against the internal time-base that can be locked to the low noise $100 \mathrm{MHz}$ output of a hydrogen maser. This event timer was originally developed for satellite laser ranging $[8,9]$.

\section{Conclusion}

Measurements of time delays are often reported without a correct uncertainty estimation. The measurements of time delays are affected by errors due to the nature of 1PPS signals, their distortion during propagation in the cables and non-ideal TICs. When the target accuracy of a measurement is better than $1 \mathrm{~ns}$, the use of a differential technique such as the pivot method is required, to allow for the mitigation of several errors that are common to both measurements. However, some of the contributions to the total uncertainty are dependent on the shape of the pulses, especially their rise time and are not mitigated by the use of the pivot technique. For pulses with similar rise times, the biggest contribution to the uncertainty is due to the TIC non-linearity and noise. For dissimilar pulses, one of which has a significantly slower rise time, the dominant factors are instead the TIC input filter bandwidth and the additional cable delays. In particular, for the widely used TIC A, the total uncertainty can be better than 100 ps when measuring similar fast-rising time pulses, but it increases to 500 ps when 
the effects of long cables and input bandwidth on dissimilar pulses (Case B) are not corrected for (see table 2).

Moreover, if the measurements are performed using TICs with wider input bandwidths, like TIC B and C, it is clear that the total uncertainty is dominated by the effect of long cables in presence of dissimilar pulses, causing the overall uncertainty to increase by one order of magnitude between Case A and Case B, from 28 ps to 227 ps if the effect of a long cable is not corrected for.

Short and low-attenuation cables are therefore highly recommended for high-accuracy time difference measurements between 1PPS signals.

We have presented some examples to serve as guidelines for a more complete estimation of the total uncertainty of measurements of time differences between dissimilar 1PPS signals available in a time metrology laboratory.

In the periodic relative calibration of time links [10] between $\mathrm{UTC}(\mathrm{k})$ laboratories it is necessary to measure the difference between the internal time base of the traveling equipment and the local representation of the UTC $(\mathrm{k})$ taking into account that the 1PPS signals can be different and located at some distance. This paper was motivated by these needs, but it will also be very useful for the periodic calibration of data acquisition systems that perform regular measurement of the time difference between the clocks of a laboratory against the local UTC(k). This paper will also support the estimation of the uncertainty budget of time differences in the context of industrial measurements and in the inter-comparisons organized by the Regional Metrology Organizations.

\section{References}

[1] JCGM100: 2008 Guide to the Expression of Uncertainty in Measurement GUM 1995 with minor corrections (JCGM in the name of the BIPM, IEC, IFCC, ILAC, ISO, IUPAC, IUPAP, and OIML) (https://www.bipm.org/utils/common/ documents/jcgm/JCGM_100_2008_E.pdf)

[2] Siccardi M, Abgrall M and Rovera G D 2012 About time measurements European Frequency and Time Forum pp $379-82$

[3] Horowitz P and Hill W 2015 The Art of Electronics 3rd edn (Cambridge: Cambridge University Press)

[4] Rovera G D, Bize S, Chupin B, Guéna J, Laurent P, Rosenbusch P, Uhrich P and Abgrall M 2016 UTC(OP) based on LNE-SYRTE atomic fountain primary frequency standards Metrologia 53 S81

[5] Siccardi M, Rovera G D and Romisch S 2016 Delay measurements of pps signals in timing systems 2016 IEEE Int. Frequency Control Symp. pp 1-6

[6] Kalisz J 2004 Review of methods for time interval measurements with picosecond resolution Metrologia 4117

[7] Rovera G, Abgrall M and Siccardi M 2012 Characterization of an auxiliary offset generator for steering of $\mathrm{H}$ Masers 26th European Frequency and Time Forum EFTF pp 14-5

[8] Samain E 2002 An ultra stable event timer Proc. of the 13th Int. Workshop on Laser Ranging Instrumentation

[9] Samain E, Exertier P, Courde C, Fridelance P, Guillemot P, Laas-Bourez M and Torre J M 2015 Time transfer by laser link: a complete analysis of the uncertainty budget Metrologia 52423

[10] Rovera G D, Torre J M, Sherwood R, Abgrall M, Courde C, Laas-Bourez M and Uhrich P 2014 Link calibration against receiver calibration: an assessment of GPS time transfer uncertainties Metrologia $\mathbf{5 1} 476$ 